\title{
Multiscale Analysis on the Pricing of Intensity-Based Defaultable Bonds
}

\author{
Sun-Hwa Cho, ${ }^{1}$ Jeong-Hoon Kim, ${ }^{1}$ and Yong-Ki $\mathrm{Ma}^{2}$ \\ ${ }^{1}$ Department of Mathematics, Yonsei University, Seoul 120-749, Republic of Korea \\ ${ }^{2}$ Department of Applied Mathematics, Kongju National University, Gongju 314-701, Republic of Korea
}

Correspondence should be addressed to Jeong-Hoon Kim; jhkim96@yonsei.ac.kr

Received 4 February 2013; Accepted 19 April 2013

Academic Editor: Alberto Cabada

Copyright (C) 2013 Sun-Hwa Cho et al. This is an open access article distributed under the Creative Commons Attribution License, which permits unrestricted use, distribution, and reproduction in any medium, provided the original work is properly cited.

This paper studies the pricing of intensity-based defaultable bonds where the volatility of default intensity is assumed to be random and driven by two different factors varying on fast and slow time scales. Corrections to the constant intensity of default are obtained and then how these corrections influence the term structure of interest rate derivatives is shown. The results indicate that the fast scale correction produces a more significant impact on the bond price than the slow scale correction and the impact tends to increase as time to maturity increases.

\section{Introduction}

In finance, the payoff for a defaultable bond would be less than the promised amount when the risky asset of a firm may default. The simplest type of the defaultable bond can be modeled by defining the time of default as the first arrival time of a Poisson process with a constant mean arrival rate called intensity. However, it becomes a common sense nowadays that the default intensity should be treated as a stochastic process depending on the macroeconomic environment. Refer to Jarrow and Protter [1], Duffie and Singleton [2], and Bielecki and Rutkowski [3] for general reference on default intensity. See also Jarrow and Turnbull [4], Lando [5], Schonbucher [6] and Musiela and Rutkowski [7] for major mathematical developments in default modeling.

This paper considers a stochastic intensity model of which motivation is described as follows. There is a recent paper by Liang et al. [8] that studied the limitation of methods for pricing credit derivatives under a reduced form framework if the default intensity process follows the Vasicek model [9]. In fact, the intensity given by the Vasicek model could be negative, whereas it should not be the case. So, this work adopts the Cox-Ingersoll-Ross (CIR) model [10] for the intensity of default. On the other hand, there are studies by B. Kim and J.-H. Kim [11] and Papageorgiou and Sircar [12] presenting the pricing of defaultable derivatives under a diffusion model for the default intensity. These works are based upon multiscale stochastic volatility described by Fouque et al. [13], where volatility follows both fast and slow scale variations. In particular, the authors of [12] showed an empirical evidence of the existence of two different scales by the calibration of the model on corporate yield curves. However, these papers consider a Vasicek model for the interest rate which severely limits the practical applications of the results since the interest rate is always positive. So, we take both the underlying interest rate and the intensity of default as CIR models whose solutions are always positive. In this sense, our model is a fundamental extension of the aforementioned models.

Also, as shown in [14], modeling the intensity of default in terms of a Cox process turned out to be inappropriate for producing loss distributions to capture real data which exhibits a heavier tail. However, as stated by Papageorgiou and Sircar [15] on multiname credit derivatives, the introduction of multiscale stochastic volatility in the default intensity process is enough to allow for a heavier tail in the loss distribution, which is compatible with empirical evidence from real data and also offsets the need for jump characteristics and maintains closed-form expressions for the conditional loss distribution. Given this observation, the volatility of the CIR model for default intensity is assumed to 
be given by a function of two different time-scale stochastic factors. The main concern of this paper is to investigate how these factors influence the interest rate derivatives.

The remaining sections are organized as follows. In Section 2, dynamics of a defaultable bond are formulated in terms of a system of multiscale stochastic differential equations of the CIR type and transformed into an asymptotic partial differential equation. In Section 3, the solution of it is approximated by using asymptotic analysis and subsequently the convergence error is estimated. Section 4 studies, numerically, the impact of the two scale factors in the stochastic intensity of default on the price and the subsequent yield curve of the bond. Section 5 is devoted to the pricing of a European option for a defaultable bond. Final remarks are given in Section 6.

\section{Problem Formulation}

In terms of short rate $r_{t}$, intensity $\lambda_{t}$, two small positive parameters $\epsilon$ and $\delta$, and two processes $Y_{t}$ and $Z_{t}$ representing a fast scale factor and a slow scale factor of the volatility of the intensity $\lambda_{t}$, respectively, we assume that dynamics of the joint process $\left(r_{t}, \lambda_{t}, Y_{t}, Z_{t}\right)$ are given by the following stochastic differential equations (SDEs):

$$
\begin{gathered}
d r_{t}=a\left(r^{*}-r_{t}\right) d t+\sigma \sqrt{r_{t}} d W_{t}^{r}, \\
d \lambda_{t}=\widehat{a}\left(\lambda^{*}-\lambda_{t}\right) d t+f\left(Y_{t}, Z_{t}\right) \sqrt{\lambda_{t}} d W_{t}^{\lambda}, \\
d Y_{t}=\frac{1}{\epsilon}\left(m-Y_{t}\right) d t+\frac{\nu \sqrt{2}}{\sqrt{\epsilon}} d W_{t}^{y}, \\
d Z_{t}=\delta g\left(Z_{t}\right) d t+\sqrt{\delta} h\left(Z_{t}\right) d W_{t}^{z}
\end{gathered}
$$

under a risk-neutral probability measure (or equivalent martingale measure), where $W_{t}^{r}, W_{t}^{\lambda}, W_{t}^{y}$, and $W_{t}^{z}$ are standard Brownian motions with a correlation structure given by the coefficients $\rho_{r \lambda}, \rho_{r y}, \rho_{r z}, \rho_{\lambda y}, \rho_{\lambda z}$, and $\rho_{y z}$ with $\rho_{r \lambda}=0$. The function $f: \mathbb{R}^{2} \rightarrow \mathbb{R}^{+}$is assumed to be bounded, smooth, and strictly positive and the functions $g$ and $h$ satisfy the Lipschitz and growth conditions so that the corresponding SDE admits a unique strong solution. We note particularly that the process $Y_{t}$ is an ergodic process whose invariant distribution is given by the Gaussian probability distribution function as

$$
\phi(y)=\frac{1}{\sqrt{2 \pi v^{2}}} \exp \left(-\frac{(y-m)^{2}}{2 v^{2}}\right), \quad y \in \mathbb{R},
$$

which provides an important averaging tool for the unobserved process $Y_{t}$ as documented well in [13]. Notation $\langle\cdot\rangle$ is going to be used for the expectation with respect to this invariant distribution.
Based on Lando [5], the price of zero-recovery defaultable bond is given by the reduced form

$$
\begin{aligned}
P^{\epsilon, \delta}( & t, r, \lambda, y, z ; T) \\
& =E^{*}\left[e^{-\int_{t}^{T}\left(r_{s}+\lambda_{s}\right) d s} \mid r_{t}=r, \lambda_{t}=\lambda, Y_{t}=y, Z_{t}=z\right]
\end{aligned}
$$

under the risk-neutral probability measure. Here, the Markov property of the joint process $\left(r_{t}, \lambda_{t}, Y_{t}, Z_{t}\right)$ was used. Of course, the zero-recovery assumption is not appropriate from an economic point of view but, for the purpose of mathematical simplicity, we assume in this paper that the loss rate equals one identically. Then, using the four-dimensional FeynmanKac formula (cf. [16]), we obtain a singularly and regularly perturbed partial differential equation (PDE) problem given by

$$
\begin{gathered}
\mathscr{L}^{\epsilon, \delta} P^{\epsilon, \delta}(t, r, \lambda, y, z ; T)=0, \quad t<T, \\
\mathscr{L}^{\epsilon, \delta}:=\frac{1}{\epsilon} \mathscr{L}_{0}+\frac{1}{\sqrt{\epsilon}} \mathscr{L}_{1}+\mathscr{L}_{2}+\sqrt{\delta} \mathscr{K}_{1}+\delta \mathscr{K}_{2}+\sqrt{\frac{\delta}{\epsilon}} \mathscr{K}_{3}, \\
\left.P^{\epsilon, \delta}(t, r, \lambda, y, z ; T)\right|_{t=T}=1,
\end{gathered}
$$

where

$$
\begin{aligned}
\mathscr{L}_{0}= & (m-y) \frac{\partial}{\partial y}+v^{2} \frac{\partial^{2}}{\partial y^{2}}, \\
\mathscr{L}_{1}= & \sqrt{2} \sigma v \rho_{r y} \sqrt{r} \frac{\partial^{2}}{\partial r \partial y}+\sqrt{2} f(y, z) v \rho_{\lambda y} \sqrt{\lambda} \frac{\partial^{2}}{\partial \lambda \partial y}, \\
\mathscr{L}_{2}= & \frac{\partial}{\partial t}+a\left(r^{*}-r\right) \frac{\partial}{\partial r}+\frac{1}{2} \sigma^{2} r \frac{\partial^{2}}{\partial r^{2}}+\widehat{a}\left(\lambda^{*}-\lambda\right) \frac{\partial}{\partial \lambda} \\
& +\frac{1}{2} f^{2}(y, z) \lambda \frac{\partial^{2}}{\partial \lambda^{2}}-(r+\lambda) I, \\
\mathscr{K}_{1}= & \sigma h(z) \rho_{r z} \sqrt{r} \frac{\partial^{2}}{\partial r \partial z}+f(y, z) h(z) \rho_{\lambda z} \sqrt{\lambda} \frac{\partial^{2}}{\partial \lambda \partial z}, \\
\mathscr{K}_{2}= & g(z) \frac{\partial}{\partial z}+\frac{1}{2} h^{2}(z) \frac{\partial^{2}}{\partial z^{2}}, \\
\mathscr{K}_{3}= & \sqrt{2} v h(z) \rho_{y z} \frac{\partial^{2}}{\partial y \partial z} .
\end{aligned}
$$

Note that $(1 / \epsilon) \mathscr{L}_{0}$ is the infinitesimal generator of the OU process $Y_{t}$. The operator $\mathscr{L}_{1}$ contains the mixed partial derivative due to the correlation between $r_{t}$ and $Y_{t}$ as well as between $\lambda_{t}$ and $Y_{t} \cdot \mathscr{L}_{2}$ is the operator of the canonical two-factor CIR model with volatility at the volatility level $f(y, z) . \mathscr{K}_{1}$ includes the mixed partial derivatives due to the correlation between $r_{t}$ and $Z_{t}$ and between $\lambda_{t}$ and $Z_{t} . \mathscr{K}_{2}$ is the infinitesimal generator of the process $Z_{t}$. $\mathscr{K}_{3}$ holds the mixed partial derivative due to the correlation between $Y_{t}$ and $Z_{t}$. 


\section{Multiscale Analysis}

Since it is difficult to solve the PDE problem (4) itself, we are interested in the following asymptotic expansions:

$$
\begin{aligned}
P^{\epsilon, \delta}(t, r, \lambda, y, z ; T) & =\sum_{j=0}^{\infty} \delta^{j / 2} P_{j}^{\epsilon}(t, r, \lambda, y, z ; T), \\
P_{j}^{\epsilon}(t, r, \lambda, y, z ; T) & =\sum_{i=0}^{\infty} \epsilon^{i / 2} P_{i, j}(t, r, \lambda, y, z ; T),
\end{aligned}
$$

so that $P^{\epsilon, \delta}$ is a series of the general term $\epsilon^{i / 2} \delta^{j / 2} P_{i, j}$. Plugging the expansion (6) into (4) leads to $P_{0}^{\epsilon}$ and $P_{1}^{\epsilon}$ given by the solutions of the PDEs

$$
\begin{gathered}
\left(\frac{1}{\epsilon} \mathscr{L}_{0}+\frac{1}{\sqrt{\epsilon}} \mathscr{L}_{1}+\mathscr{L}_{2}\right) P_{0}^{\epsilon}(t, r, \lambda, y, z ; T)=0, \quad t<T, \\
\left.P_{0}^{\epsilon}(t, r, \lambda, y, z ; T)\right|_{t=T}=1, \\
\left(\frac{1}{\epsilon} \mathscr{L}_{0}+\frac{1}{\sqrt{\epsilon}} \mathscr{L}_{1}+\mathscr{L}_{2}\right) P_{1}^{\epsilon}(t, r, \lambda, y, z ; T) \\
=-\left(\mathscr{K}_{1}+\frac{1}{\sqrt{\epsilon}} \mathscr{K}_{3}\right) P_{0}^{\epsilon}, \quad t<T \\
\left.P_{1}^{\epsilon}(t, r, \lambda, y, z ; T)\right|_{t=T}=0,
\end{gathered}
$$

respectively. From now on, we employ an analytic technique of [13] to approximate the two terms $P_{0}^{\epsilon}$ and $P_{1}^{\epsilon}$ based on the ergodic property of the Ornstein-Uhlenbeck (OU) process $Y_{t}$.

3.1. The Leading Order Term. Applying the expansion (7) with $j=0$ to (8) leads to

$$
\begin{aligned}
\frac{1}{\epsilon} \mathscr{L}_{0} P_{0,0} & +\frac{1}{\sqrt{\epsilon}}\left(\mathscr{L}_{0} P_{1,0}+\mathscr{L}_{1} P_{0,0}\right) \\
& +\left(\mathscr{L}_{0} P_{2,0}+\mathscr{L}_{1} P_{1,0}+\mathscr{L}_{2} P_{0,0}\right) \\
& +\sqrt{\epsilon}\left(\mathscr{L}_{0} P_{3,0}+\mathscr{L}_{1} P_{2,0}+\mathscr{L}_{2} P_{1,0}\right) \\
& +\cdots=0
\end{aligned}
$$

from which we obtain an affine form of the leading order term $P_{0,0}$ as follows.

Theorem 1. Assume that the partial derivative of $P_{i, j}$ with respect to $y$ does not grow as much as $\partial P_{i, j} / \partial y \sim e^{y^{2} / 2}$ as $y$ goes to infinity. Then the leading order term $P_{0}:=P_{0,0}$ of the expansion (7) with $j=0$ is independent of the fast scale variable $y$ and further it has the affine representation

$$
P_{0}(T-\tau, r, \lambda, z ; T)=\breve{C}(\tau, z) e^{-r \breve{A}(\tau)-\lambda \breve{B}(\tau)}
$$

with $\tau=T-t, \breve{A}(0)=\breve{B}(0)=0$ and $\breve{C}(0, z)=1$, where $\breve{A}(\tau)$, $\breve{B}(\tau)$, and $\breve{C}(\tau, z)$ are given by,

$$
\begin{gathered}
\breve{A}(\tau)=\frac{2\left(e^{\gamma_{1} \tau}-1\right)}{2 \gamma_{1}+\left(\gamma_{1}+a\right)\left(e^{\gamma_{1} \tau}-1\right)}, \\
\breve{B}(\tau)=\frac{2\left(e^{\gamma_{2} \tau}-1\right)}{2 \gamma_{2}+\left(\gamma_{2}+\widehat{a}\right)\left(e^{\gamma_{2} \tau}-1\right)}, \\
\breve{C}(\tau, z)=\left[\frac{2 \gamma_{1} e^{(1 / 2)\left(\gamma_{1}+a\right) \tau}}{2 \gamma_{1}+\left(\gamma_{1}+a\right)\left(e^{\gamma_{1} \tau}-1\right)}\right]^{2 a r^{*} / \sigma^{2}} \\
\times\left[\frac{2 \gamma_{2} e^{(1 / 2)\left(\gamma_{2}+\widehat{a}\right) \tau}}{2 \gamma_{2}+\left(\gamma_{2}+\widehat{a}\right)\left(e^{\gamma_{2} \tau}-1\right)}\right]^{2 \widehat{a} \lambda^{*} / \breve{\sigma}^{2}(z)}, \\
\gamma_{1}:=\sqrt{a^{2}+2 \sigma^{2}}, \quad \gamma_{2}:=\sqrt{\widehat{a}^{2}+2 \breve{\sigma}^{2}(z)},
\end{gathered}
$$

respectively. Here, $\breve{\sigma}$ is defined by

$$
\breve{\sigma}^{2}(z):=\left\langle f^{2}\right\rangle(z)=\int_{\mathbb{R}} f^{2}(y, z) \phi(y) d y
$$

in terms of $\phi$ (the invariant distribution of $Y_{t}$ ).

Proof. Multiplying (10) by $\epsilon$ and letting $\epsilon$ go to zero, we obtain the ordinary differential equation (ODE) $\mathscr{L}_{0} P_{0}=0$. Recalling that the operator $\mathscr{L}_{0}$ is the generator of the OU process $Y_{t}$, the solution $P_{0}$ of this ODE must be independent of the $y$ variable due to the assumed growth condition; $P_{0}=$ $P_{0}(t, r, \lambda, z ; T)$. From the $O(1 / \sqrt{\epsilon})$ terms of $(10)$, we have the PDE $\mathscr{L}_{0} P_{1,0}+\mathscr{L}_{1} P_{0}=0$. If we apply the $y$-independence of $P_{0}$ to this PDE, then it reduces to the ODE $\mathscr{L}_{0} P_{1,0}=0$ and so $P_{1,0}$ is independent of $y$ by the same reason as in the case of $P_{0} ; P_{1,0}=P_{1,0}(t, r, \lambda, z ; T)$. So far, the two terms $P_{0}$ and $P_{1,0}$ do not depend on the current level $y$ of the fast scale volatility driving the process $Y_{t}$.

One can continue to eliminate the terms of order $1, \sqrt{\epsilon}$, $\epsilon$, and so forth. From the $O(1)$ terms of (10), we get $\mathscr{L}_{0} P_{2,0}+$ $\mathscr{L}_{1} P_{1,0}+\mathscr{L}_{2} P_{0}=0$. This PDE becomes

$$
\mathscr{L}_{0} P_{2,0}+\mathscr{L}_{2} P_{0}=0
$$

due to the $y$-independence of $P_{1,0}$ obtained above. Equation (14) is a Poisson equation for $P_{2,0}$ with respect to the operator $\mathscr{L}_{0}$ in the $y$ variable. It is well known (cf. [17]) from the Fredholm alternative (solvability condition) that it has a solution only if $\mathscr{L}_{2} P_{0}$ is centered with respect to the invariant distribution of $Y_{t}$; that is,

$$
\begin{aligned}
\left\langle\mathscr{L}_{2}\right\rangle P_{0}= & 0, \\
\left\langle\mathscr{L}_{2}\right\rangle:= & \frac{\partial}{\partial t}+a\left(r^{*}-r\right) \frac{\partial}{\partial r}+\frac{1}{2} \sigma^{2} r \frac{\partial^{2}}{\partial r^{2}}+\widehat{a}\left(\lambda^{*}-\lambda\right) \frac{\partial}{\partial \lambda} \\
& +\frac{1}{2} \breve{\sigma}^{2}(z) \lambda \frac{\partial^{2}}{\partial \lambda^{2}}-(r+\lambda) I .
\end{aligned}
$$

Noting that (15) is nothing but a PDE corresponding to the intensity with constant volatility replaced by the function $\breve{\sigma}(z)$, the affine solution of (15) is given by (11)-(12) from the well-known solution in [6]. 
3.2. The Correction Terms. Next, we derive the first-order correction terms $P_{1,0}$ and $P_{0,1}$ from the leading order solution $P_{0}$ obtained above. Contrary to the result obtained in [12], the correction terms are not given by closed form solutions in the present case of a CIR model (instead of a Vasicek model) for the interest rate.

Theorem 2. Assume that the partial derivative of $P_{i, j}$ with respect to $y$ does not grow as much as $\partial P_{i, j} / \partial y \sim e^{y^{2} / 2}$ as $y$ goes to infinity. The correction term $P_{1,0}$ is independent of the variable $y$ and $\widetilde{P}_{1,0}^{\epsilon}:=\sqrt{\epsilon} P_{1,0}(t, r, \lambda, z ; T)$ is given by

$$
\widetilde{P}_{1,0}^{\epsilon}(T-\tau, r, \lambda, z ; T)=\breve{D}(\tau, r, \lambda, z) P_{0}(T-\tau, r, \lambda, z ; T)
$$

with $\breve{D}(0, r, \lambda, z)=0$, where $\breve{D}(\tau, r, \lambda, z)$ is given by the solution of the PDE

$$
\begin{gathered}
\frac{\partial \breve{D}}{\partial \tau}-\left\{a\left(r^{*}-r\right)-\sigma^{2} r \breve{A}(\tau)\right\} \frac{\partial \breve{D}}{\partial r}-\frac{1}{2} \sigma^{2} r \frac{\partial^{2} \breve{D}}{\partial r^{2}} \\
-\left\{\widehat{a}\left(\lambda^{*}-\lambda\right)-\breve{\sigma}^{2}(z) \lambda \breve{B}(\tau)\right\} \frac{\partial \breve{D}}{\partial \lambda} \\
-\frac{1}{2} \breve{\sigma}^{2}(z) \lambda \frac{\partial^{2} \breve{D}}{\partial \lambda^{2}}=l(\tau, r, \lambda, z), \\
l(\tau, r, \lambda, z):=q(z) \sqrt{\lambda} \breve{B}^{3}(\tau)-s(z) \sqrt{r} \breve{A}(\tau) \breve{B}^{2}(\tau), \\
q(z):=\frac{\nu \sqrt{\epsilon}}{\sqrt{2}} \rho_{\lambda y} \sqrt{\lambda}\left\langle f \theta_{y}\right\rangle, \\
s(z):=\frac{\nu \sqrt{\epsilon}}{\sqrt{2}} \rho_{r y} \sigma \sqrt{r}\left\langle\theta_{y}\right\rangle .
\end{gathered}
$$

Here, $\breve{A}(\tau)$ and $\breve{B}(\tau)$ are given by (12) and the function $\theta$ : $\mathbb{R}^{2} \rightarrow \mathbb{R}$ is defined by the solution of

$$
\mathscr{L}_{0} \theta(y, z)=f^{2}(y, z)-\left\langle f^{2}\right\rangle(z)
$$

and $\theta_{y}$ denotes the partial derivative with respect to the $y$ variable.

Proof. The $O(\sqrt{\epsilon})$ terms of $(10)$ lead to $\mathscr{L}_{0} P_{3,0}+\mathscr{L}_{1} P_{2,0}+$ $\mathscr{L}_{2} P_{1,0}=0$ which is a Poisson equation for $P_{3,0}$ and so the solvability condition leads to the PDE

$$
\left\langle\mathscr{L}_{1} P_{2,0}+\mathscr{L}_{2} P_{1,0}\right\rangle=0 .
$$

Meanwhile, from (14) and (15), we have

$$
P_{2,0}=-\mathscr{L}_{0}^{-1}\left(\mathscr{L}_{2}-\left\langle\mathscr{L}_{2}\right\rangle\right) P_{0}+c(t, r, \lambda, z)
$$

for some function $c(t, r, \lambda, z)$ that does not rely on the $y$ variable. Plugging (20) into (19), a PDE for $P_{1,0}$ is obtained as

$$
\left\langle\mathscr{L}_{2}\right\rangle P_{1,0}=\left\langle\mathscr{L}_{1} \mathscr{L}_{0}^{-1}\left(\mathscr{L}_{2}-\left\langle\mathscr{L}_{2}\right\rangle\right)\right\rangle P_{0}
$$

with the terminal condition $\left.P_{1,0}(t, r, \lambda, z ; T)\right|_{t=T}=0$. This implies that $P_{1,0}$ is $y$-independent. Since we focus on the firstorder correction terms to $P_{0}$, we reset (21) with respect to $\widetilde{P}_{1,0}^{\epsilon}$ leading to

$$
\begin{gathered}
\left\langle\mathscr{L}_{2}\right\rangle \widetilde{P}_{1,0}^{\epsilon}=\mathscr{H}_{1} P_{0}, \\
\mathscr{H}_{1}:=\sqrt{\epsilon}\left\langle\mathscr{L}_{1} \mathscr{L}_{0}^{-1}\left(\mathscr{L}_{2}-\left\langle\mathscr{L}_{2}\right\rangle\right)\right\rangle .
\end{gathered}
$$

To calculate the operator $\mathscr{H}_{1}$, we first find

$$
\mathscr{L}_{2}-\left\langle\mathscr{L}_{2}\right\rangle=\frac{1}{2}\left(f^{2}(y, z)-\left\langle f^{2}\right\rangle(z)\right) \frac{\partial^{2}}{\partial \lambda^{2}}
$$

from (5) and so the operator $\mathscr{H}_{1}$ is expressed as $\mathscr{H}_{1}=$ $q(z) \sqrt{\lambda} \partial_{\lambda \lambda \lambda}^{3}+s(z) \sqrt{r} \partial_{r \lambda \lambda}^{3}$, where $q(z)$ and $s(z)$ are the ones given in the theorem. Then, from (15), Theorem 1 and the change of variables $\tau=T-t$, the PDE (22) leads to

$$
\begin{aligned}
\frac{\partial \widetilde{P}_{1,0}^{\epsilon}=}{\partial \tau}= & a\left(r^{*}-r\right) \frac{\partial \widetilde{P}_{1,0}^{\epsilon}}{\partial r}+\frac{1}{2} \sigma^{2} r \frac{\partial^{2} \widetilde{P}_{1,0}^{\epsilon}}{\partial r^{2}}+\widehat{a}\left(\lambda^{*}-\lambda\right) \frac{\partial \widetilde{P}_{1,0}^{\epsilon}}{\partial \lambda} \\
& +\frac{1}{2} \breve{\sigma}^{2}(z) \lambda \frac{\partial^{2} \widetilde{P}_{1,0}^{\epsilon}}{\partial \lambda^{2}}-(r+\lambda) \widetilde{P}_{1,0}^{\epsilon} \\
& +\breve{C}(\tau, z) e^{-r \breve{A}(\tau)-\lambda \breve{B}(\tau)} \\
& \times\left(q(z) \sqrt{\lambda} \breve{B}^{3}(\tau)+s(z) \sqrt{r} \breve{A}(\tau) \breve{B}^{2}(\tau)\right)
\end{aligned}
$$

with the initial condition $\left.\widetilde{P}_{1,0}^{\epsilon}(T-\tau, r, \lambda, z ; T)\right|_{\tau=0}=0$. Finally, plugging $\widetilde{P}_{1,0}^{\epsilon}$ of the form (16) into (24), we obtain the result of Theorem 2 by direct computation. follows.

Similarly, one can derive the correction term $P_{0,1}$ also as

Theorem 3. Assume that the partial derivative of $P_{i, j}$ with respect to $y$ does not grow as much as $\partial P_{i, j} / \partial y \sim e^{y^{2} / 2}$ as $y$ goes to infinity. The first correction term $P_{0,1}$ does not depend on the variable $y$ and $\widetilde{P}_{0,1}^{\delta}:=\sqrt{\delta} P_{0,1}(t, r, \lambda, z ; T)$ is given by

$$
\widetilde{P}_{0,1}^{\delta}(T-\tau, r, \lambda, z ; T)=\breve{E}(\tau, r, \lambda, z) P_{0}(T-\tau, r, \lambda, z ; T)
$$

with $\breve{E}(0, r, \lambda, z)=0$, where $\breve{E}(\tau, r, \lambda, z)$ is given by the solution of the PDE

$$
\begin{aligned}
\frac{\partial \breve{E}}{\partial \tau}- & \left\{a\left(r^{*}-r\right)-\sigma^{2} r \breve{A}(\tau)\right\} \frac{\partial \breve{E}}{\partial r}-\frac{1}{2} \sigma^{2} r \frac{\partial^{2} \breve{E}}{\partial r^{2}} \\
& -\left\{\widehat{a}\left(\lambda^{*}-\lambda\right)-\breve{\sigma}^{2}(z) \lambda \breve{B}(\tau)\right\} \frac{\partial \breve{E}}{\partial \lambda} \\
& -\frac{1}{2} \breve{\sigma}^{2}(z) \lambda \frac{\partial^{2} \breve{E}}{\partial \lambda^{2}}=m(\tau, r, \lambda, z), \\
m(\tau, r, \lambda, z): & =\frac{1}{\breve{C}}\left(u(z) \sqrt{r} \breve{A}(\tau) \frac{\partial \breve{C}}{\partial z}+v(z) \sqrt{\lambda} \breve{B}(\tau) \frac{\partial \breve{C}}{\partial z}\right),
\end{aligned}
$$




$$
\begin{gathered}
u(z):=-\sqrt{\delta} \rho_{r z} \sigma h(z), \\
v(z):=-\sqrt{\delta} \rho_{\lambda z} \bar{\sigma}(z) h(z) .
\end{gathered}
$$

Here, $\breve{A}(\tau), \breve{B}(\tau)$, and $\breve{C}(\tau, z)$ are given by (12) and $\bar{\sigma}$ is defined by

$$
\bar{\sigma}(z):=\langle f\rangle(z)=\int_{\mathbb{R}} f(y, z) \phi(y) d y
$$

in terms of $\phi$ (the invariant distribution of $Y_{t}$ ).

Proof. Applying the expansion (7) with $j=0$ and $j=1$ to (9) results in

$$
\begin{aligned}
\frac{1}{\epsilon} \mathscr{L}_{0} P_{0,1} & +\frac{1}{\sqrt{\epsilon}}\left(\mathscr{L}_{0} P_{1,1}+\mathscr{L}_{1} P_{0,1}\right) \\
& +\left(\mathscr{L}_{0} P_{2,1}+\mathscr{L}_{1} P_{1,1}+\mathscr{L}_{2} P_{0,1}\right) \\
& +\sqrt{\epsilon}\left(\mathscr{L}_{0} P_{3,1}+\mathscr{L}_{1} P_{2,1}+\mathscr{L}_{2} P_{1,1}\right) \\
& +\cdots \\
= & -\frac{1}{\sqrt{\epsilon}} \mathscr{K}_{3} P_{0}-\left(\mathscr{K}_{1} P_{0}+\mathscr{K}_{3} P_{1,0}\right) \\
& -\sqrt{\epsilon}\left(\mathscr{K}_{1} P_{1,0}+\mathscr{K}_{3} P_{2,0}\right)+\cdots .
\end{aligned}
$$

Then, from the $O(1 / \epsilon)$ term of $(28), P_{0,1}$ is $y$-independent as the solution of the ODE $\mathscr{L}_{0} P_{0,1}=0$ under the assumed growth condition. Since both $P_{0}$ (obtained in Theorem 1) and $P_{0,1}$ are $y$-independent, from the $O(1 / \sqrt{\epsilon})$ terms of (28), we have the ODE $\mathscr{L}_{0} P_{1,1}=0$. So, by the same reason as in the case of $P_{0,1}=0, P_{1,1}$ is also independent of $y$. Hence, the two terms $P_{0,1}$ and $P_{1,1}$ do not depend on the current level $y$ of the fast scale volatility of intensity.

One can continue to remove the terms of order $1, \sqrt{\epsilon}, \epsilon$, and so forth. From the $O(1)$ terms and the $y$-independence of $P_{1,0}$ and $P_{1,1}$, we have the PDE $\mathscr{L}_{0} P_{2,1}+\mathscr{L}_{2} P_{0,1}+\mathscr{K}_{1} P_{0}=0$. This is a Poisson equation for $P_{2,1}$ with respect to the operator $\mathscr{L}_{0}$ in the $y$ variable and so it has a solution only if $\left\langle\mathscr{L}_{2}\right\rangle P_{0,1}=$ $-\left\langle\mathscr{K}_{1}\right\rangle P_{0}$ holds. If we reset this $\mathrm{PDE}$ with respect to $\widetilde{P}_{0,1}^{\delta}$, then we have

$$
\begin{aligned}
& \left\langle\mathscr{L}_{2}\right\rangle \widetilde{P}_{0,1}^{\delta}=\mathscr{H}_{2} P_{0}, \\
& \mathscr{H}_{2}:=-\sqrt{\delta}\left\langle\mathscr{K}_{1}\right\rangle,
\end{aligned}
$$

where the operator $\mathscr{H}_{2}$ is the same as $\mathscr{H}_{2}=u(z) \sqrt{r} \partial_{r z}^{2}+$ $v(z) \sqrt{\lambda} \partial_{\lambda z}^{2}$ from the definition of $\mathscr{K}_{1}$ given by (5).
Using the operator (15), Theorem 1, and the change of variable $\tau=T-t$, we write the PDE (29) as follows:

$$
\begin{aligned}
\frac{\partial \widetilde{P}_{0,1}^{\delta}}{\partial \tau}= & a\left(r^{*}-r\right) \frac{\partial \widetilde{P}_{0,1}^{\delta}}{\partial r}+\frac{1}{2} \sigma^{2} r \frac{\partial^{2} \widetilde{P}_{0,1}^{\delta}}{\partial r^{2}}+\widehat{a}\left(\lambda^{*}-\lambda\right) \frac{\partial \widetilde{P}_{0,1}^{\delta}}{\partial \lambda} \\
& +\frac{1}{2} \breve{\sigma}^{2}(z) \lambda \frac{\partial^{2} \widetilde{P}_{0,1}^{\delta}}{\partial \lambda^{2}}-(r+\lambda) \widetilde{P}_{0,1}^{\delta} \\
& +e^{-r \breve{A}(\tau)-\lambda \breve{B}(\tau)}\left(u(z) \sqrt{r} \breve{A}(\tau) \frac{\partial \breve{C}}{\partial z}\right. \\
& \left.+v(z) \sqrt{\lambda} \breve{B}(\tau) \frac{\partial \breve{C}}{\partial z}\right)
\end{aligned}
$$

where the initial condition $\left.\widetilde{P}_{0,1}^{\delta}(T-\tau, r, \lambda, z ; T)\right|_{\tau=0}=0$ is satisfied. Plugging the form (25) into (30), we obtain the result of Theorem 3 by direct computation.

3.3. Accuracy. Synthesizing Theorems 1, 2, and 3, we obtain the following asymptotic representation of the price of the defaultable bond at time $t$ :

$$
\begin{aligned}
P^{\epsilon, \delta}(t, r, \lambda, y, z ; T) \approx & \widetilde{P}^{\epsilon, \delta}:=P_{0}+\widetilde{P}_{1,0}^{\epsilon}+\widetilde{P}_{0,1}^{\delta} \\
= & (1+\breve{D}(\tau, r, \lambda, z)+\breve{E}(\tau, r, \lambda, z)) \\
& \times \breve{C}(\tau, z) e^{-r \breve{A}(\tau)-\lambda \breve{B}(\tau)},
\end{aligned}
$$

where $\breve{A}(\tau), \breve{B}(\tau), \breve{C}(\tau, z), \breve{D}(\tau, r, \lambda, z)$, and $\breve{E}(\tau, r, \lambda, z)$ are given by (12), (17), and (3), respectively. We note that the function $g(z)$ (the drift term of the slow scale variation) in (1) does not affect the present form of the approximated bond price $\widetilde{P}^{\epsilon, \delta}$ due to the order of $\delta$ in front of $g(z)$ in (1). It should influence the next order approximation.

In this approximation, the leading order price is determined by the functions $\breve{A}(\tau), \breve{B}(\tau)$, and $\breve{C}(\tau, z)$ and the firstorder corrections are given by $\breve{D}(\tau, r, \lambda, z)$ and $\breve{E}(\tau, r, \lambda, z)$. Here, $\breve{D}(\tau, r, \lambda, z)$ is determined by the two group parameters $q(z)$ and $s(z)$ and $\breve{E}(\tau, r, \lambda, z)$ is determined by $u(z)$ and $v(z)$. These four group parameters absorb the original parameters and functions related to $Y_{t}$ and $Z_{t}$. The group parameter $q(z)$ absorbs $\epsilon, \nu, \rho_{\lambda y}$, and $f$ while $s(z)$ absorbs $\epsilon, \nu, \rho_{r y}$, and $f$. The group parameter $u(z)$ absorbs $\delta, \rho_{r z}$, and $h$ while $v(z)$ absorbs $\delta, \rho_{\lambda z}$, and $h$. Particularly, the functions $f, g$ and $h$ determining the original model (1) need not be specified to price the defaultable bond. The asymptotic expansions based upon the ergodic property of the OU process $Y_{t}$ provide the reduction of the model parameters and the absorption of the model functions into the group parameters.

All the derivations given so far are formal (as usually done in this kind of research work). So, we discuss the accuracy of the asymptotic approximation (31) as shown in the following theorem. 
Theorem 4. Let $\widetilde{P}^{\epsilon, \delta}$ be defined by (31). Then for any fixed $(t, r, \lambda, y, z)$ there exists a positive constant $C$, which depends on $(t, r, \lambda, y, z)$ but not on $\epsilon$ and $\delta$, such that

$$
P^{\epsilon, \delta}(t, r, \lambda, y, z ; T)-\widetilde{P}^{\epsilon, \delta}(t, r, \lambda, z ; T) \leq C(\epsilon+\delta)
$$

holds for all $0<\epsilon, \delta \leq 1$.

Proof. To prove the inequality (32), we first define a residual $R^{\epsilon, \delta}$ by writing $P^{\epsilon, \delta}$ as

$$
P^{\epsilon, \delta}=\sum_{i, j=0}^{i, j=3} \epsilon^{i / 2} \delta^{j / 2} P_{i, j}-R^{\epsilon, \delta} .
$$

The second step is to compute $\mathscr{L}^{\epsilon, \delta} R^{\epsilon, \delta}$ using the properties of $P^{\epsilon, \delta}, P_{0}, \ldots, P_{3,3}$ obtained above. The last step is to write $R^{\epsilon, \delta}$ as a probabilistic representation (the Feynman-Kac formula) and to show the desired estimate (32). This type of argument is standard and our formulation would not make a different impact on the derivation in Theorem 3.1 of [12]. So, we leave the details there.

\section{Numerical Experiment}

In this section, we compute numerically the fast term factor $\breve{D}$ and the slow term factor $\breve{E}$ of the stochastic volatility of default intensity and investigate the impact of the multiscale factors on the functional behavior of the bond price with the constant volatility of default intensity.

Table 1 shows the average cumulative default rates of bond issuers for the period of 1983-2009. It says, for instance, that over a ten-year $B$-rated issuers were in default at a $44.982 \%$ average rate between 1983 and 2009. Using this table, in general, one can calculate the average default intensity between time 0 and $t$ from the cumulative probability of default by time $t$. For example, the average 20 -year default intensity for $B$-rated issuers is given by $-(1 / 20) \ln (1-0.65493)=0.0532$.

Based on Table 1 and the data [13], we choose the parameter values $a=1.2, \widehat{a}=1.3, \sigma=0.06, \breve{\sigma}^{2}=0.03, r^{*}=$ $0.1, \lambda=0.0455$, and $\lambda^{*}=0.0532$. Maturity runs from 0 to 20 years and the interest rate is fixed as $r=0.07$. Figures 1 and 2 show the bond price correction term structure depending on the group parameters $(q, s)$ and $(u, v)$, respectively, for the fast term factor $\breve{D}$ and the slow term factor $\breve{E}$. One can notice that the fast scale correction creates a more significant impact on the bond price than the slow scale correction and the impact tends to increase as time to maturity increases.

\section{Bond Option Pricing}

In this section, we are interested in an asymptotic pricing formula for a European option, where the underlying asset itself is a zero-coupon bond with default risk.

We use notations $T$ and $T_{0}, T_{0}<T$, to denote the maturity of the defaultable bond and the maturity of a European option written on the defaultable bond, respectively. It is assumed that the option becomes invalid when a default occurs prior

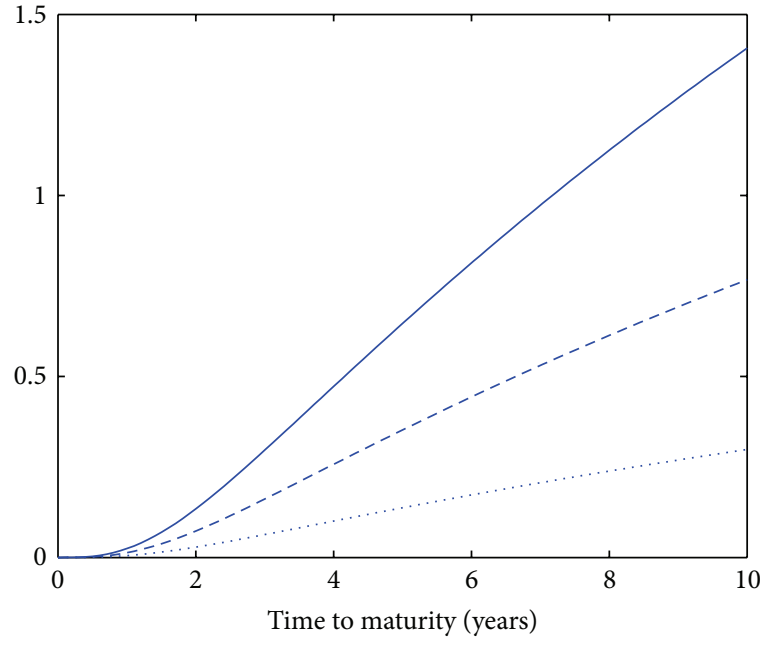

FIGURE 1: The price correction term structure factor $\breve{D}$ is shown by dotted, dashed, and sold lines as functions of time to maturity, respectively, for $(q, s)=(0.2,0.1),(0.3,0.4)$, and $(0.6,0.7)$.

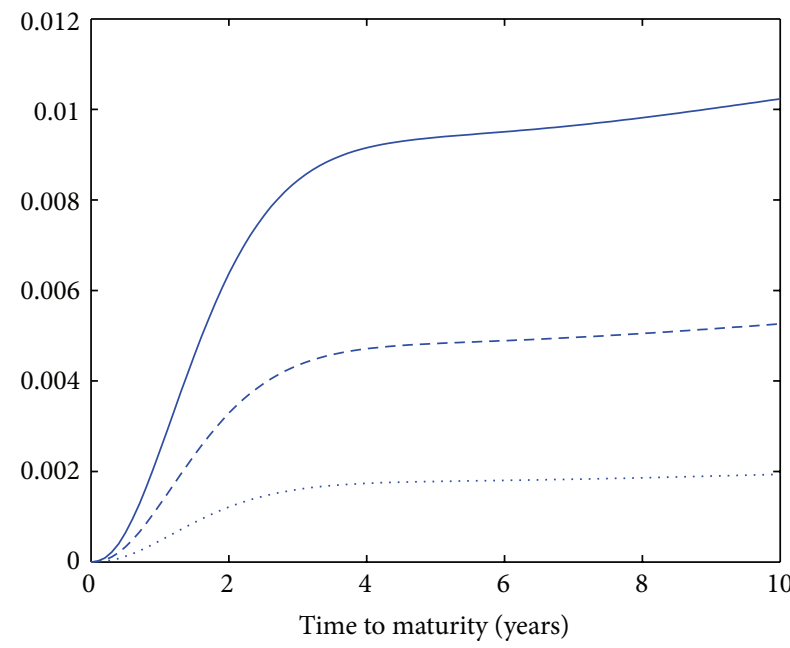

FIgURE 2: The price correction term structure factor $\breve{E}$ is shown by dotted, dashed and sold lines as functions of time to maturity, respectively, for $(u, v)=(0.1,0.2),(0.3,0.5)$, and $(0.7,0.8)$.

to $T_{0}$. The short rate process $r_{t}$, the intensity process $\lambda_{t}$, the fast volatility process $Y_{t}$ of the intensity, and the slow volatility process $Z_{t}$ of the intensity are given by the SDEs (1).

The option price at time $t$ for an observed short rate $r_{t}=r$, an intensity level $\lambda_{t}=\lambda$, a fast volatility level $Y_{t}=y$, and a slow volatility level $Z_{t}=z$, denoted by $Q\left(t, r, \lambda, y, z ; T_{0}, T\right)$, is defined by

$$
\begin{aligned}
& Q\left(t, r, \lambda, y, z ; T_{0}, T\right) \\
& =E^{*}\left[e^{-\int_{t}^{T_{0}} r_{s} d s} h\left(\mathscr{B}\left(T_{0}, T\right)\right) \mid r_{t}=r, \lambda_{t}=\lambda, Y_{t}=y, Z_{t}=z\right]
\end{aligned}
$$


TABLE 1: Average cumulative default rates (\%), 1983-2009 (source: Moody's).

\begin{tabular}{lccccccc}
\hline Rating & Year 1 & Year 2 & Year 5 & Year 7 & Year 10 & Year 15 & Year 20 \\
\hline Aaa & 0 & 0.016 & 0.086 & 0.182 & 0.187 & 0.187 & 0.187 \\
Aa & 0.024 & 0.066 & 0.247 & 0.318 & 0.408 & 0.989 & 3.583 \\
A & 0.057 & 0.187 & 0.806 & 1.297 & 2.099 & 6.536 \\
Baa & 0.196 & 0.543 & 2.027 & 3.130 & 4.815 & 8.719 & 12.603 \\
Ba & 1.209 & 3.434 & 11.444 & 15.600 & 21.128 & 30.467 & 38.152 \\
B & 4.550 & 10.519 & 26.24 & 35.054 & 44.982 & 57.136 & 65.493 \\
Caa & 15.383 & 26.969 & 50.339 & 57.783 & 71.993 & 82.434 & 86.669 \\
Ca-C & 36.207 & 48.440 & 70.176 & 74.757 & 78.014 & 78.014 & - \\
All rated & 1.761 & 3.620 & 8.046 & 9.991 & 12.100 & 14.990 & 18.002 \\
\hline
\end{tabular}

under an equivalent martingale measure, where the bond price $\mathscr{B}\left(T_{0}, T\right)$ is given by

$$
\begin{aligned}
\mathscr{B}\left(T_{0}, T\right) & =P^{\epsilon, \delta}\left(T_{0}, r_{T_{0}}, \lambda_{T_{0}}, Y_{T_{0}}, Z_{T_{0}} ; T\right) \\
& =E^{*}\left[e^{-\int_{T_{0}}^{T}\left(r_{s}+\lambda_{s}\right) d s} \mid r_{T_{0}}, \lambda_{T_{0}}, Y_{T_{0}}, Z_{T_{0}}\right]
\end{aligned}
$$

and $h$ is the payoff function of the option at time $T_{0}$. Here, it is assumed that the payoff function $h$ is at best linearly growing at infinity and is a smooth function. This smoothness assumption may be too severe in practical point of view as $h$ is not differentiable at the exercise price in the case of classical European call or put option. The smoothness assumption on $h$, however, can be removed similarly to the argument in [18] or [12]. So, for simplicity, $h$ is assumed to be a smooth function.

By the four-dimensional Feynman-Kac formula, we transform the above integral form (34) into the PDE

$$
\begin{aligned}
\frac{\partial Q}{\partial t}+ & a\left(r^{*}-r\right) \frac{\partial Q}{\partial r}+\frac{1}{2} \sigma^{2} r \frac{\partial^{2} Q}{\partial r^{2}}+\widehat{a}\left(\lambda^{*}-\lambda\right) \frac{\partial Q}{\partial \lambda} \\
& +\frac{1}{2} f^{2}(y, z) \lambda \frac{\partial^{2} Q}{\partial \lambda^{2}} \\
& +\frac{1}{\epsilon}(m-y) \frac{\partial Q}{\partial y}+\frac{\nu^{2}}{\epsilon} \frac{\partial^{2} Q}{\partial y^{2}}+\delta g(z) \frac{\partial Q}{\partial z} \\
& +\frac{1}{2} \delta h^{2}(z) \frac{\partial^{2} Q}{\partial z^{2}} \\
& +\rho_{r y} \sigma \sqrt{r} \frac{\nu \sqrt{2}}{\sqrt{\epsilon}} \frac{\partial^{2} Q}{\partial r \partial y}+\rho_{r z} \sigma \sqrt{r} \sqrt{\delta} h(z) \frac{\partial^{2} Q}{\partial r \partial z} \\
& +\rho_{\lambda y} f(y, z) \sqrt{\lambda} \frac{\nu \sqrt{2}}{\sqrt{\epsilon}} \frac{\partial^{2} Q}{\partial \lambda \partial y} \\
& +\rho_{\lambda z} f(y, z) \sqrt{\lambda} \sqrt{\delta} h(z) \frac{\partial^{2} Q}{\partial \lambda \partial z} \\
& +\rho_{y z} \frac{\nu \sqrt{2}}{\sqrt{\epsilon}} \sqrt{\delta} h(z) \frac{\partial^{2} Q}{\partial y \partial z}-(r+\lambda) Q=0
\end{aligned}
$$

with the terminal condition $\left.Q\left(t, r, \lambda, y, z ; T_{0}, T\right)\right|_{t=T_{0}}=$ $h\left(P^{\epsilon, \delta}\left(T_{0}, r, \lambda, y, z ; T\right)\right)$. Then, keeping the notation used in
Section 3 but with a different terminal condition, the option price $Q\left(t, r, \lambda, y, z ; T_{0}, T\right)$ is given by the solution of the PDE problem

$$
\begin{gathered}
\mathscr{L}^{\epsilon, \delta} Q\left(t, r, \lambda, y, z ; T_{0}, T\right)=0, \\
\left.Q\left(t, r, \lambda, y, z ; T_{0}, T\right)\right|_{t=T_{0}}=h\left(P^{\epsilon, \delta}\left(T_{0}, r, \lambda, y, z ; T\right)\right) .
\end{gathered}
$$

Taking the asymptotic expansions

$$
\begin{gathered}
Q=Q_{0}^{\epsilon}+\sqrt{\delta} Q_{1}^{\epsilon}+\delta Q_{2}^{\epsilon}+\cdots, \\
Q_{k}^{\epsilon}=Q_{0, k}+\sqrt{\epsilon} Q_{1, k}+\epsilon Q_{2, k}+\epsilon^{3 / 2} Q_{3, k}+\cdots, \\
k=0,1,2, \ldots, \\
\left.Q\left(t, r, \lambda, y, z ; T_{0}, T\right)\right|_{t=T_{0}}=h\left(P_{0}\left(T_{0}, r, \lambda, z ; T\right)\right) \\
+\widetilde{P}_{1,0}^{\epsilon}\left(T_{0}, r, \lambda, z ; T\right) h^{\prime}\left(P_{0}\left(T_{0}, r, \lambda, z ; T\right)\right) \\
+\widetilde{P}_{0,1}^{\delta}\left(T_{0}, r, \lambda, z ; T\right) h^{\prime}\left(P_{0}\left(T_{0}, r, \lambda, z ; T\right)\right) \\
+\cdots,
\end{gathered}
$$

where $P_{0}, \widetilde{P}_{1,0}^{\epsilon}$, and $\widetilde{P}_{0,1}^{\delta}$ are given by Theorems 1,2 , and 3 , respectively, we employ the same asymptotic analysis as in Section 3 to derive an asymptotic pricing formula for the bond option.

First, the terms of order $1 / \epsilon$ and $1 / \sqrt{\epsilon}$ in the asymptotic $\operatorname{PDE}$ (37) yield the $y$-independence of $Q_{0,0}$ (in brief, $\left.Q_{0}\right), Q_{1,0}$, and $Q_{0,1}$ under the same growth condition as in Section 3 .

The $O(1)$ terms in (37) give a Poisson equation for $Q_{2,0}$ from which the solvability condition $\left\langle\mathscr{L}_{2}\right\rangle Q_{0}=0$ has to be satisfied. From (40), the corresponding terminal condition is given by $\left.Q_{0}\right|_{t=T_{0}}=h\left(P_{0}\left(T_{0}, r, \lambda, z ; T\right)\right)$. Then we have

$$
\begin{aligned}
& Q_{0}\left(t, r, \lambda, z ; T_{0}, T\right) \\
& =E^{*}\left[e^{-\int_{t}^{T_{0}} r_{s} d s} h\left(P_{0}\left(T_{0}, r, \lambda, z ; T\right)\right) \mid r_{t}=r, \lambda_{t}=\lambda, Z_{t}=z\right],
\end{aligned}
$$

where $P_{0}\left(T_{0}, r, \lambda, z ; T\right)$ is given by (11) in Theorem 1 at time $t=T_{0}$. 
The $O(\sqrt{\epsilon})$ terms in (37) lead to a Poisson equation for $Q_{3,0}$, where the solvability condition is given by $\left\langle\mathscr{L}_{1} Q_{2,0}+\right.$ $\left.\mathscr{L}_{2} Q_{1,0}\right\rangle=0$. If we put $\widetilde{Q}_{1,0}:=\sqrt{\epsilon} Q_{1,0}$, then this solvability condition leads to

$$
\left\langle\mathscr{L}_{2}\right\rangle \widetilde{Q}_{1,0}\left(t, r, \lambda, z ; T_{0}, T\right)=\mathscr{H}_{1} Q_{0}\left(t, r, \lambda, z ; T_{0}, T\right)
$$

with a terminal condition given by

$$
\begin{aligned}
\widetilde{Q}_{1,0} & \left.\left(t, r, \lambda, z ; T_{0}, T\right)\right|_{t=T_{0}} \\
& =\widetilde{P}_{1,0}^{\epsilon}\left(T_{0}, r, \lambda, z ; T\right) h^{\prime}\left(P_{0}\left(T_{0}, r, \lambda, z ; T\right)\right)
\end{aligned}
$$

from (40), where the operator $\mathscr{H}_{1}$ is given by (22) and $\widetilde{P}_{1,0}^{\epsilon}$ is given by the solution (16) in Theorem 2 . Then, by applying the Feynman-Kac formula to (42) and (43), we obtain the following probabilistic representation:

$$
\begin{gathered}
\widetilde{Q}_{1,0}\left(t, r, \lambda, z ; T_{0}, T\right) \\
=E^{*}\left[e^{-\int_{t}^{T_{0}} r_{s} d s} \widetilde{P}_{1,0}^{\epsilon}\left(T_{0}, r, \lambda, z ; T\right) h^{\prime}\left(P_{0}\left(T_{0}, r, \lambda, z ; T\right)\right)\right. \\
\quad-\int_{t}^{T_{0}} e^{-\int_{t}^{u} r_{s} d s} \mathscr{H}_{1} Q_{0}\left(u, r, \lambda, z ; T_{0}, T\right) d u \mid r_{t}=r, \\
\left.\lambda_{t}=\lambda, Z_{t}=z\right] .
\end{gathered}
$$

Now, the $O(\sqrt{\delta})$ terms in (37) lead to a Poisson equation for $Q_{2,1}$ such that the solvability condition $\left\langle\mathscr{L}_{2} Q_{0,1}\right\rangle=-\left\langle\mathscr{K}_{1} Q_{0}\right\rangle$ holds. If we let $\widetilde{Q}_{0,1}:=\sqrt{\delta} Q_{0,1}$, then this solvability condition leads to

$$
\left\langle\mathscr{L}_{2}\right\rangle \widetilde{Q}_{0,1}\left(t, r, \lambda, z ; T_{0}, T\right)=\mathscr{H}_{2} Q_{0}\left(t, r, \lambda, z ; T_{0}, T\right),
$$

where the operator $\mathscr{H}_{2}$ is given by (29). Also, from the terminal condition (40), we have

$$
\begin{aligned}
\widetilde{Q}_{0,1} & \left.\left(t, r, \lambda, z ; T_{0}, T\right)\right|_{t=T_{0}} \\
& =\widetilde{P}_{0,1}^{\delta}\left(T_{0}, r, \lambda, z ; T\right) h^{\prime}\left(P_{0}\left(T_{0}, r, \lambda, z ; T\right)\right),
\end{aligned}
$$

where $\widetilde{P}_{0,1}^{\delta}$ is given by the solution (25) in Theorem 3. By applying the Feynman-Kac formula to (45) and (46), we have the following probabilistic representation:

$$
\begin{aligned}
\widetilde{Q}_{0,1}\left(t, r, \lambda, z ; T_{0}, T\right) \\
=E^{*}\left[e^{-\int_{t}^{T_{0}} r_{s} d s} \widetilde{P}_{0,1}^{\delta}\left(T_{0}, r, \lambda, z ; T\right) h^{\prime}\left(P_{0}\left(T_{0}, r, \lambda, z ; T\right)\right)\right. \\
\quad-\int_{t}^{T_{0}} e^{-\int_{t}^{u} r_{s} d s} \mathscr{H}_{2} Q_{0}\left(u, r, \lambda, z ; T_{0}, T\right) d u \mid r_{t}=r, \\
\left.\quad \lambda_{t}=\lambda, Z_{t}=z\right] .
\end{aligned}
$$

Synthesizing the above results, we obtain an asymptotic pricing formula for a European option written on the defaultable bond as follows.
Theorem 5. The option price $Q\left(t, r, \lambda, y, z ; T_{0}, T\right)$ defined by (34)-(35) is given by

$$
\begin{aligned}
Q\left(t, r, \lambda, y, z ; T_{0}, T\right) \approx & Q_{0}\left(t, r, \lambda, z ; T_{0}, T\right) \\
& +\widetilde{Q}_{1,0}\left(t, r, \lambda, z ; T_{0}, T\right) \\
& +\widetilde{Q}_{0,1}\left(t, r, \lambda, z ; T_{0}, T\right),
\end{aligned}
$$

where $Q_{0}, \widetilde{Q}_{1,0}$, and $\widetilde{Q}_{0,1}$ are given by (41), (44), and (47), respectively.

\section{Conclusion}

Based upon a reduced form framework of credit risk, this paper investigates the term structure of interest rate derivatives by utilizing an asymptotic expansion method. Mainly, it is focused on the multiscale stochastic volatility of default intensity of the CIR type. Firstly, we obtain an approximation to the value of the defaultable bond as an extension of the known affine solution for the constant volatility. A small correction to the case of the constant volatility has a useful feature that the original model parameters and functions are replaced by the four group parameters. They melt into the group parameters in the averaged form by the ergodic property of the fast mean-reverting OU process, which is a desirable outcome for the purpose of calibration in practical applications. Secondly, we find out the dependence structure of the two scale factors of the stochastic volatility on the bond price and subsequent yield curve, which suggests some flexibility of the multifactor intensity model. This paper, however, has not tested the model to prove this flexibility from empirical evaluation. Furthermore, it is necessary to illustrate the performance of the model setup for the fitting of yield curves. The model calibration and empirical evidence are left to future extensions which also include the pricing of the credit default swap (CDS) spread as opposed to studies based on a Cox process such as [19]. Furthermore, it should be interesting to apply the framework of this paper to multiname intensity models for the pricing of collateralized debt obligation (CDO).

\section{Acknowledgment}

The authors thank the anonymous referees for helpful remarks to improve this paper. This study was supported by the National Research Foundation of Korea NRF-20100008717.

\section{References}

[1] R. A. Jarrow and P. Protter, "Structural versus reduced form models: a new information based perspective," Journal of Investment Management, vol. 2, no. 2, pp. 1-10, 2004.

[2] D. Duffie and K. J. Singleton, Credit Risk, Princeton University Press, 2003.

[3] T. R. Bielecki and M. Rutkowski, Credit Risk: Modelling, Valuation and Hedging, Springer, New York, NY, USA, 2002. 
[4] R. A. Jarrow and S. M. Turnbull, "Pricing derivatives on financial securities subject to credit risk," Journal of Finance, vol. 50, no. 1, pp. 53-85, 1995.

[5] D. Lando, "On Cox processes and credit-risky securities," Review of Derivatives Research, vol. 2, no. 2-3, pp. 99-120, 1998.

[6] P. J. Schonbucher, "Term structure modelling of defaultable bonds," Review of Derivatives Research, vol. 2, no. 2-3, pp. 161192, 1998.

[7] M. Musiela and M. Rutkowski, Martingale Methods in Financial Modelling, Springer, New York, NY, USA, 2nd edition, 2007.

[8] J. Liang, J. M. Ma, T. Wang, and Q. Ji, "Valuation of portfolio credit derivatives with default intensities using the Vasicek model," Asia-Pacific Financial Markets, vol. 18, no. 1, pp. 33-54, 2011.

[9] O. Vasicek, "An equilibrium characterization of the term structure," Journal of Financial Economics, vol. 5, no. 2, pp. 177-188, 1977.

[10] J. C. Cox, J. E. Ingersoll Jr., and S. A. Ross, "A theory of the term structure of interest rates," Econometrica, vol. 53, no. 2, pp. 385407, 1985.

[11] B. Kim and J.-H. Kim, "Default risk in interest rate derivatives with stochastic volatility," Quantitative Finance, vol. 11, no. 12, pp. 1837-1845, 2011.

[12] E. Papageorgiou and R. Sircar, "Multiscale intensity models for single name credit derivatives," Applied Mathematical Finance, vol. 15, no. 1-2, pp. 73-105, 2008.

[13] J.-P. Fouque, G. Papanicolaou, R. Sircar, and K. Sølna, Multiscale Stochastic Volatility for Equity, Interest Rate, and Credit Derivatives, Cambridge University Press, Cambridge, UK, 2011.

[14] A. Mortensen, "Semi-analytical valuation of basket credit derivatives in intensity-based models," Journal of Derivatives, vol. 13, no. 4, pp. 8-26, 2006.

[15] E. Papageorgiou and R. Sircar, "Multiscale intensity models and name grouping for valuation of multi-name credit derivatives," Applied Mathematical Finance, vol. 16, no. 3-4, pp. 353-383, 2009.

[16] B. Oksendal, Stochastic Differential Equations, Springer, New York, NY, USA, 6th edition, 2003.

[17] A. G. Ramm, "A simple proof of the Fredholm alternative and a characterization of the Fredholm operators," The American Mathematical Monthly, vol. 108, no. 9, pp. 855-860, 2001.

[18] J.-P. Fouque, G. Papanicolaou, R. Sircar, and K. Solna, "Multiscale stochastic volatility asymptotics," SIAM Journal on Multiscale Modeling and Simulation, vol. 2, no. 1, pp. 22-42, 2003.

[19] Y.-K. Ma and J.-H. Kim, "Pricing the credit default swap rate for jump diffusion default intensity processes," Quantitative Finance, vol. 10, no. 8, pp. 809-817, 2010. 


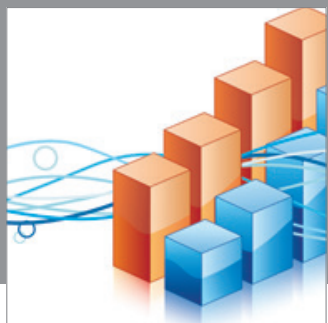

Advances in

Operations Research

mansans

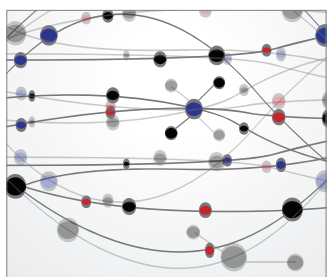

The Scientific World Journal
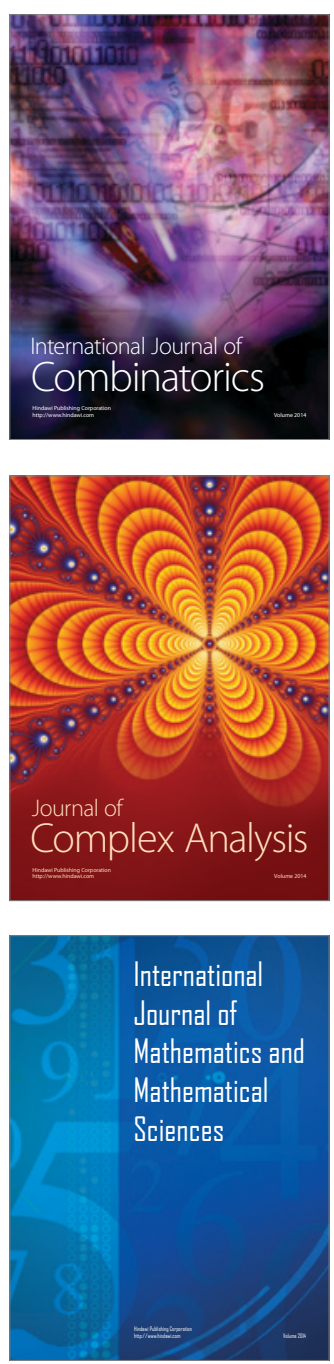
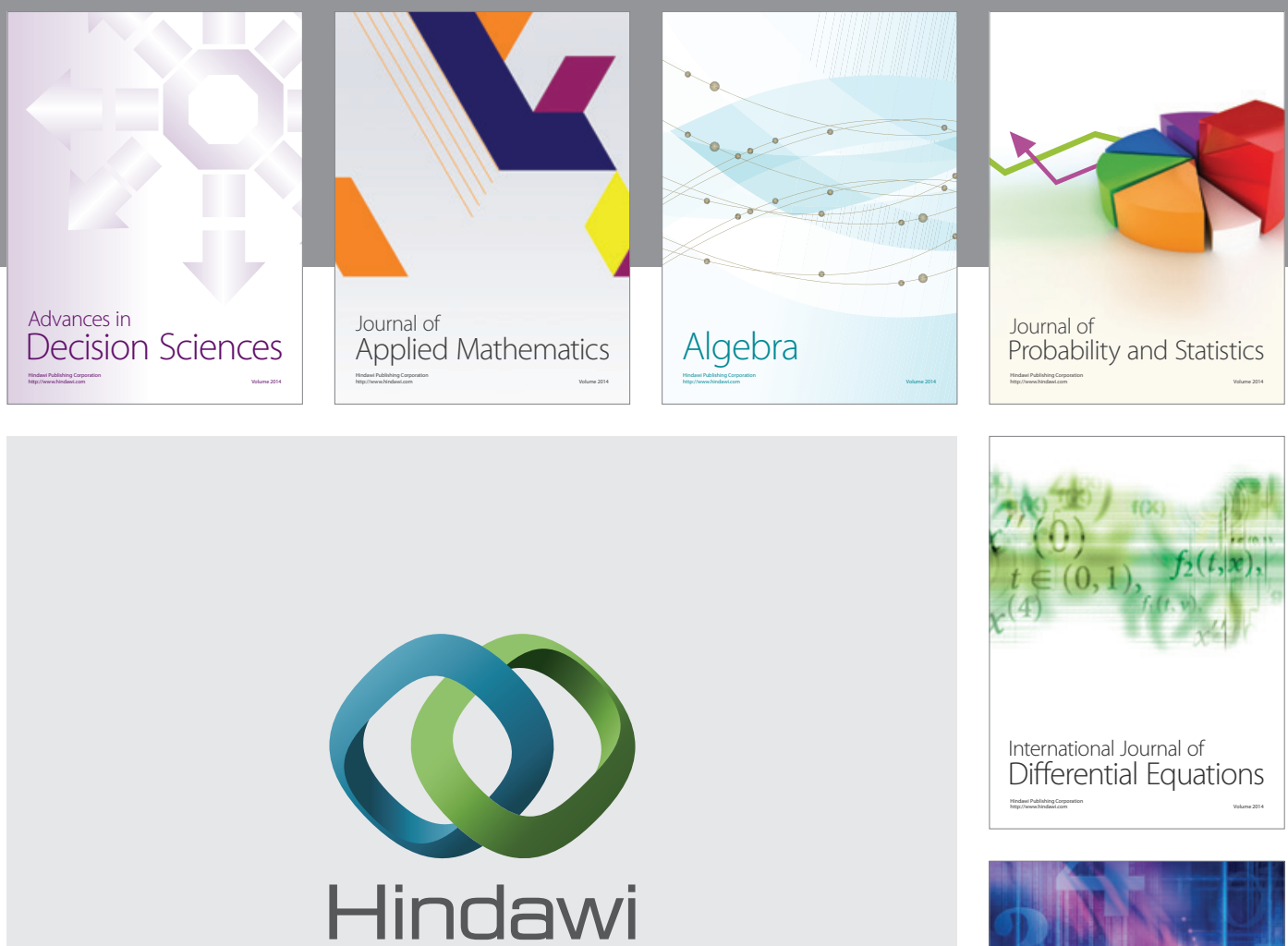

Submit your manuscripts at http://www.hindawi.com
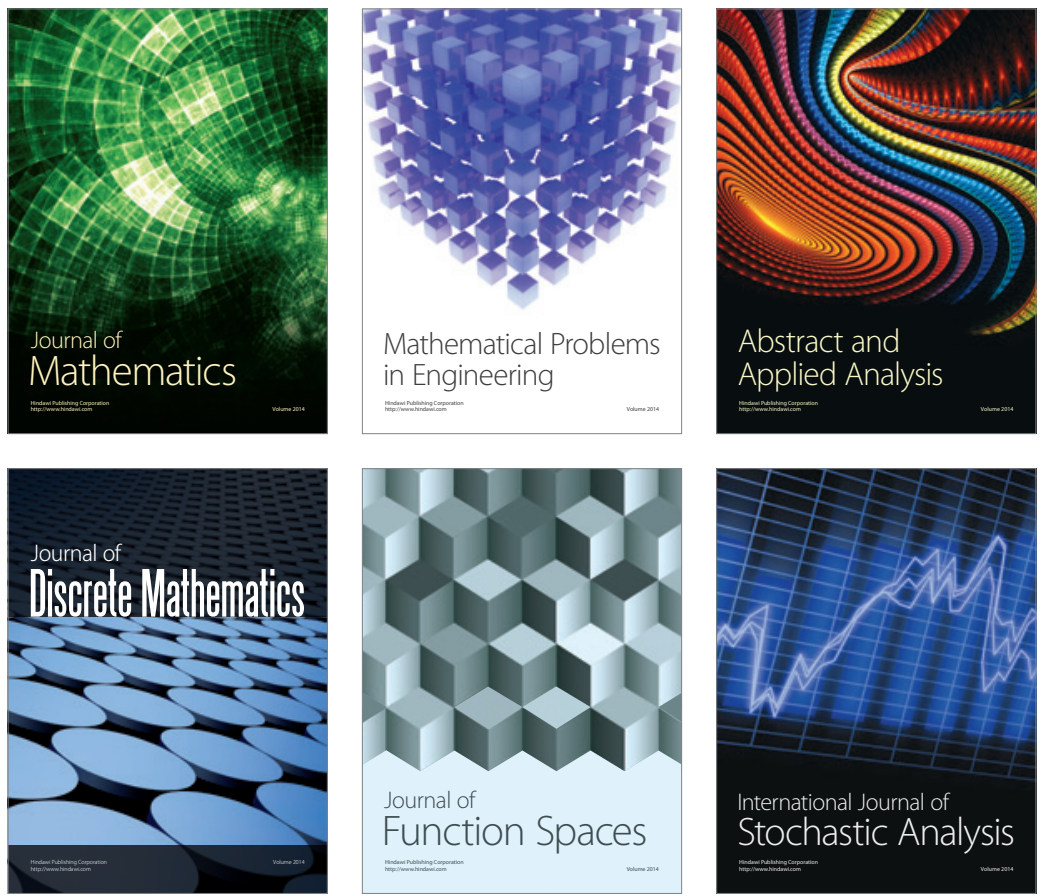

Journal of

Function Spaces

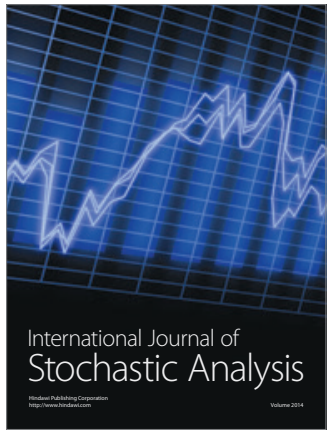

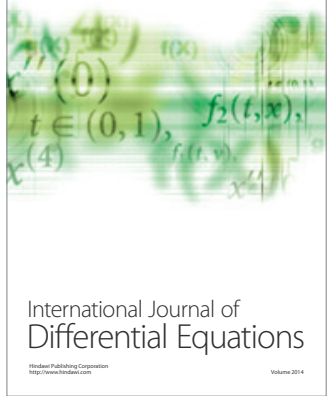
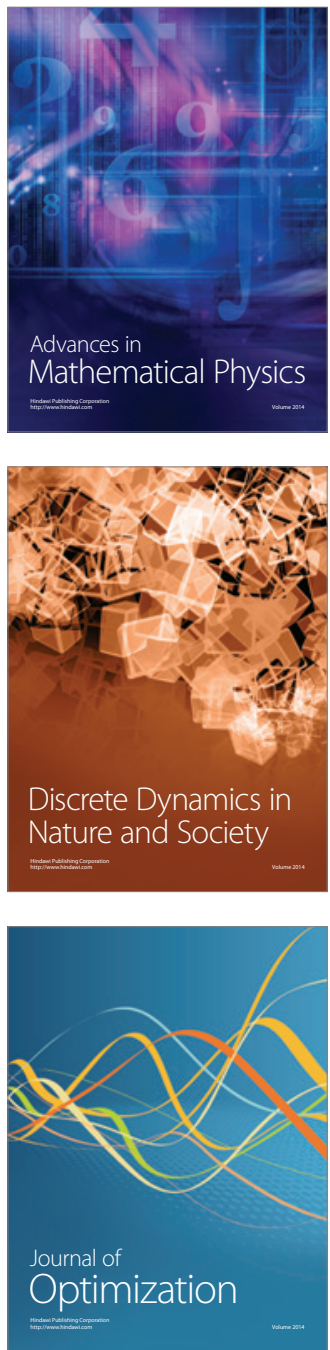